\title{
Oxaliplatin, Fluorouracil, and Leucovorin as Adjuvant Treatment for Colon Cancer
}

\author{
Thierry André, M.D., Corrado Boni, M.D., Lamia Mounedji-Boudiaf, M.D., \\ Matilde Navarro, M.D., Josep Tabernero, M.D., Tamas Hickish, M.D., \\ Clare Topham, M.D., Marta Zaninelli, M.D., Philip Clingan, M.D., \\ John Bridgewater, M.D., Isabelle Tabah-Fisch, M.D., \\ and Aimery de Gramont, M.D., for the Multicenter International Study \\ of Oxaliplatin/5-Fluorouracil/Leucovorin in the Adjuvant Treatment \\ of Colon Cancer (MOSAIC) Investigators
}

\begin{abstract}
The standard adjuvant treatment of colon cancer is fluorouracil plus leucovorin (FL). Oxaliplatin improves the efficacy of this combination in patients with metastatic colorectal cancer. We evaluated the efficacy of treatment with FL plus oxaliplatin in the postoperative adjuvant setting.

METHODS

We randomly assigned 2246 patients who had undergone curative resection for stage II or III colon cancer to receive FL alone or with oxaliplatin for six months. The primary end point was disease-free survival.
\end{abstract}

RESULTS

A total of 1123 patients were randomly assigned to each group. After a median followup of 37.9 months, 237 patients in the group given FL plus oxaliplatin had had a cancer-related event, as compared with 293 patients in the FL group (21.1 percent vs. 26.1 percent; hazard ratio for recurrence, $0.77 ; \mathrm{P}=0.002$ ). The rate of disease-free survival at three years was 78.2 percent ( 95 percent confidence interval, 75.6 to 80.7 ) in the group given FL plus oxaliplatin and 72.9 percent (95 percent confidence interval, 70.2 to 75.7 ) in the FL group ( $\mathrm{P}=0.002$ by the stratified log-rank test). In the group given FL plus oxaliplatin, the incidence of febrile neutropenia was 1.8 percent, the incidence of gastrointestinal adverse effects was low, and the incidence of grade 3 sensory neuropathy was 12.4 percent during treatment, decreasing to 1.1 percent at one year of follow-up. Six patients in each group died during treatment (death rate, 0.5 percent).

CONCLUSIONS

Adding oxaliplatin to a regimen of fluorouracil and leucovorin improves the adjuvant treatment of colon cancer.
From Hôpital Tenon, Paris (T.A.); GERCOR Oncology Multidisciplinary Group, Paris (T.A., A.G.); Arcispedale Santa Maria Nuova, Reggio Emilia, Italy (C.B.); Sanofi-Synthelabo, Paris (L.M.-B., I.T.-F.); Hospital Duran i Reynals, l'Hospitalet de Llobregat, Llobregat, Spain (M.N.); Vall d'Hebron University Hospital, Barcelona, Spain (J.T.); Dorset Cancer Centre, Royal Bournemouth and Poole Hospitals, Bournemouth, United Kingdom (T.H.); Royal Surrey County Hospital, Guildford, Surrey, United Kingdom (C.T.); Ospedale Borgotrento, Verona, Italy (M.Z.); Southern Medical Day Care Centre, Wollongong, Australia (P.C.); North Middlesex Hospital, London (J.B.); and Hôpital Saint-Antoine, Paris (A.G.). Address reprint requests to Dr. de Gramont at Hôpital Saint Antoine, 184 rue du Faubourg Saint Antoine, Paris 75012, France, or at aimery.de-gramont@ sat.ap-hop-paris.fr.

N EnglJ Med 2004;350:2343-51.

Coppright $\odot 2004$ Massachusetts Medical Society. 


\section{C}

OLORECTAL CANCER IS THE SECOND

leading cause of death from cancer in

Western countries ${ }^{1} ; 40$ to 50 percent of patients who undergo potentially curative surgery alone ultimately relapse and die of metastatic disease. ${ }^{2}$ The most important prognostic indicator of survival in early colon cancer is the stage of the tumor ( $\mathrm{T}$, according to the tumor-node-metastasis [TNM] classification), determined by the depth of penetration through the bowel wall, and the number of involved lymph nodes. ${ }^{3}$

The demonstration that postoperative adjuvant treatment with fluorouracil and levamisole reduced the mortality rate by 33 percent among patients with stage III colon cancer ${ }^{4}$ prompted several trials, which established six months of treatment with fluorouracil plus leucovorin (FL) as the standard adjuvant chemotherapy for stage III colon cancer. ${ }^{5-11}$ Oxaliplatin is a third-generation platinum derivative, which, when combined with fluorouracil and leucovorin, is among the most effective chemotherapies for metastatic colorectal cancer. ${ }^{12-15}$ To determine whether oxaliplatin can also benefit patients with disease in an earlier stage, we conducted an international phase 3 clinical trial in patients with stage II or III colon cancer - the Multicenter International Study of Oxaliplatin/5-Fluorouracil/ Leucovorin in the Adjuvant Treatment of Colon Cancer (MOSAIC).

METHODS

\section{PATIENTS}

Patients were eligible if they had undergone complete resection of histologically proven stage II (T3 or T4,N0,M0) or stage III (any T,N1 or N2,M0) colon cancer, as defined by the presence of the inferior pole of the tumor above the peritoneal reflection - that is, at least $15 \mathrm{~cm}$ from the anal margin. Treatment had to be started within seven weeks after surgery. Other eligibility criteria included an age of 18 to 75 years; a Karnofsky performance-status score of at least 60; a carcinoembryonic antigen level of less than $10 \mathrm{ng}$ per milliliter; the absence of prior chemotherapy, immunotherapy, or radiotherapy; and adequate blood counts and liver and kidney function. Written informed consent was required from all patients, and the study was approved by the ethics committees of the participating centers.

\section{TREATMENT}

Eligible patients were randomly assigned to receive FL alone or with oxaliplatin. In the FL group, each cycle comprised a 2-hour infusion of $200 \mathrm{mg}$ of leucovorin per square meter of body-surface area followed by a bolus of $400 \mathrm{mg}$ of fluorouracil per square meter and then a 22-hour infusion of 600 mg of fluorouracil per square meter given on 2 consecutive days every 14 days, for 12 cycles. In the group given FL plus oxaliplatin, the same FL regimen was used, plus a two-hour infusion of $85 \mathrm{mg}$ of oxaliplatin (Eloxatin, Sanofi-Synthelabo) per square meter on day 1 , given simultaneously with leucovorin, with the use of a $Y$ infusion device. The use of disposable pumps (LV5 infusors, Baxter Healthcare) allowed outpatients to receive a continuous infusion of fluorouracil.

Adverse effects were graded according to the Common Toxicity Criteria of the National Cancer Institute, version 1. According to these criteria, a score of 1 indicates mild adverse effects, a score of 2 moderate adverse effects, a score of 3 severe adverse effects, and a score of 4 life-threatening adverse effects. Dose reductions were based on the worst adverse effects observed during the previous cycle. The dose of oxaliplatin was to be reduced to $75 \mathrm{mg}$ per square meter in the event of persistent (at least 14 days) paresthesias, temporary painful paresthesias, or functional impairment. Oxaliplatin was discontinued in cases of persistent painful paresthesias or functional impairment. Together with reductions in the dose of oxaliplatin, the bolus dose of fluorouracil was reduced to $300 \mathrm{mg}$ per square meter and the infusion to $500 \mathrm{mg}$ per square meter in the event of grade 3 or 4 neutropenia or thrombocytopenia (or both), diarrhea, stomatitis, or other drug-related adverse effects of grade 3. Only the dose of fluorouracil was scheduled to be reduced in the event of skin-related adverse effects of grade 3 or 4 . Treatment was delayed by up to three weeks until the patient recovered from various adverse effects, the neutrophil count exceeded 1500 per cubic millimeter, and the platelet count exceeded 100,000 per cubic millimeter. Chemotherapy was stopped in the event of cardiac or neurocerebellar adverse effects or grade 3 or 4 allergic reactions.

\section{FOLLOW-UP}

Patients were assessed before randomization, every two weeks during treatment, and then every six 
months for five years. The baseline assessment involved a medical history taking, physical examination, biologic tests, measurement of the carcinoembryonic antigen level, chest radiography, and abdominal ultrasonography or computed tomography. Patients were monitored for adverse effects throughout the treatment period and until 28 days after the last cycle of chemotherapy, unless treatment-related adverse effects required additional follow-up.

The diagnosis of recurrence was made on the basis of imaging and, if necessary, cytologic analysis or biopsy. An elevated carcinoembryonic antigen level as a solitary finding was not accepted as evidence of relapse. Neurologic adverse effects were to be reported at each visit during follow-up and were assessed with the use of the neurosensory section of the Common Toxicity Criteria of the National Cancer Institute, version 1.

\section{STATISTICAL ANALYSIS}

Randomization was performed centrally, and the minimization method was used to balance treatment allocation according to the TNM stage (T2 or $\mathrm{T} 3$ vs. T4 and N0, N1, or N2), the presence or absence of bowel obstruction or tumor perforation, and the medical center. The sample size of 2200 patients was calculated under the assumptions of a three-year disease-free survival rate of 73 percent in the control group and 79 percent in the group given FL plus oxaliplatin, with a ratio of stage II disease to stage III disease of 0.4:0.6, an enrollment period and a follow-up period of three years, a decrease in the risk of relapse after three years, a statistical power of 90 percent, and an alpha value of 0.05 and two-sided $P$ values derived with the use of the log-rank test. The primary efficacy variable was disease-free survival, defined as the time from randomization to relapse or death, whichever occurred first. Second colorectal cancers were considered relapses, whereas noncolorectal tumors were disregarded in the analyses.

The primary statistical analysis of efficacy was the comparison, after three years of follow-up, of disease-free survival between groups according to the intention-to-treat principle, with the use of a two-sided log-rank test stratified according to baseline disease stage. Hazard ratios and 95 percent confidence intervals were calculated with the use of the Cox proportional-hazards model. Survival curves were drawn according to Kaplan-Meier methods.

To assess the consistency of the effect of treat- ment on disease-free survival across prognostic subgroups, we calculated hazard ratios and 95 percent confidence limits for subgroups defined according to the following variables: sex, age, disease stage (II vs. III), baseline serum carcinoembryonic antigen level, number of involved lymph nodes ( $\leq 4$ vs. $>4$ ), $\mathrm{T}$ classification (T4 vs. T1, T2, or T3), degree of cellular differentiation (well vs. poorly differentiated), and the presence or absence of perforation, obstruction, and venous invasion.

The cutoff date of the analysis was April 22, 2003. The duration of follow-up was defined as the number of months from randomization to the cutoff date.

Secondary end points were safety, including long-term adverse effects, and overall survival, measured from the time of randomization to death from any cause. With a median follow-up of three years, it is too early to compare the two treatment groups statistically in terms of survival, and only descriptive analyses of overall survival are presented. Safety analyses included patients who had received at least one cycle of treatment.

\section{ORGANIZATION OF THE TRIAL}

The concept underlying this study was developed by Dr. de Gramont, and the investigation was designed by the investigators and Sanofi-Synthelabo. Data were collected, managed, and analyzed by the sponsor. The article was written by the investigators, on the basis of data and statistical analyses provided by Sanofi-Synthelabo.

A data and safety monitoring board of independent experts reviewed safety data every six months during the treatment period to provide the sponsor with independent advice on the progress of the study and on safety. No interim analysis was planned or performed.

\section{RESULTS}

\section{STUDY POPULATION}

Between October 1998 and January 2001, 2246 patients were enrolled at 146 centers in 20 countries: 1123 patients were randomly assigned to receive FL plus oxaliplatin and 1123 to receive FL without oxaliplatin. Of these patients, 1108 received at least one cycle of FL plus oxaliplatin and 1111 received at least one cycle of FL. The patients' characteristics were well matched in the two groups (Table 1). In both groups, 60 percent of the patients had stage III disease and 40 percent had stage II disease. The 


\begin{tabular}{|c|c|c|}
\hline Characteristic & $\begin{array}{l}\text { FL plus Oxaliplatin } \\
\quad(\mathrm{N}=1123)\end{array}$ & $\begin{array}{c}\mathrm{FL} \\
(\mathrm{N}=1123)\end{array}$ \\
\hline \multicolumn{3}{|l|}{ All patients } \\
\hline \multicolumn{3}{|l|}{ Age $-\mathrm{yr}$} \\
\hline Median & 61 & 60 \\
\hline Range & 19-75 & $20-75$ \\
\hline Age $<65$ yr - no. (\%) & $723(64.4)$ & $743(66.2)$ \\
\hline \multicolumn{3}{|l|}{ Sex - no. (\%) } \\
\hline Male & $630(56.1)$ & $588(52.4)$ \\
\hline Female & $493(43.9)$ & $535(47.6)$ \\
\hline \multicolumn{3}{|c|}{ Karnofsky performance-status score - no. (\%) } \\
\hline$<60$ & $5(0.4)$ & $5(0.4)$ \\
\hline $60-70$ & $150(13.4)$ & $134(11.9)$ \\
\hline $80-100$ & $968(86.2)$ & $984(87.6)$ \\
\hline \multicolumn{3}{|l|}{ Disease stage — no. (\%) } \\
\hline II & $451(40.2)$ & 448 (39.9) \\
\hline III & $672(59.8)$ & $675(60.1)$ \\
\hline \multicolumn{3}{|l|}{ Depth of invasion - no. (\%) } \\
\hline $\mathrm{T} 2$ & $51(4.5)$ & $54(4.8)$ \\
\hline T3 & $853(76.0)$ & $852(75.9)$ \\
\hline $\mathrm{T} 4$ & $213(19.0)$ & $208(18.5)$ \\
\hline Unknown & $6(0.5)$ & $9(0.8)$ \\
\hline Bowel obstruction - no. (\%) & $201(17.9)$ & $217(19.3)$ \\
\hline Perforation - no. (\%) & $78(6.9)$ & $78(6.9)$ \\
\hline \multicolumn{3}{|l|}{ Histologic appearance — no. (\%) } \\
\hline Well differentiated & $934(83.2)$ & $914(81.4)$ \\
\hline Poorly differentiated & $142(12.6)$ & $148(13.2)$ \\
\hline Unknown & $47(4.2)$ & $61(5.4)$ \\
\hline \multicolumn{3}{|c|}{ Patients with stage III disease - no. (\%) } \\
\hline \multicolumn{3}{|l|}{ No. of nodes involved } \\
\hline $1-4$ & $499(44.4)$ & $513(45.7)$ \\
\hline$>4$ & $170(15.1)$ & $160(14.2)$ \\
\hline Unknown & $2(0.2)$ & $2(0.2)$ \\
\hline \multicolumn{3}{|c|}{ Patients with stage II disease - no. (\%) } \\
\hline $\mathrm{T} 4$ & $84(18.6)$ & $87(19.4)$ \\
\hline \multicolumn{3}{|l|}{ No. of lymph nodes examined } \\
\hline$<10$ & $152(33.7)$ & $149(33.3)$ \\
\hline$\geq 10$ & $295(65.4)$ & $294(65.6)$ \\
\hline Bowel obstruction & $71(15.7)$ & $87(19.4)$ \\
\hline Perforation & $38(8.4)$ & $43(9.6)$ \\
\hline \multicolumn{3}{|l|}{ Histologic appearance } \\
\hline Well differentiated & $385(85.4)$ & $378(84.4)$ \\
\hline Poorly differentiated & $47(10.4)$ & $42(9.4)$ \\
\hline Unknown & $19(4.2)$ & $28(6.3)$ \\
\hline
\end{tabular}

overall median time between surgery and the start of chemotherapy was 5.7 weeks (range, 1.1 to 17.0).

A total of 41 patients (1.8 percent) did not strictly meet eligibility criteria related to baseline disease. In one patient in each group, the resection of primary tumor was incomplete. Four patients in the group given FL plus oxaliplatin and six in the FL group had a history of cancer, including colorectal cancer. Thirteen patients (four in the group given FL plus oxaliplatin and nine in the FL group) had stage IV cancer, and three patients (two in the group given FL plus oxaliplatin and one in the FL group) had cancer of the middle or lower rectum. Four patients in the group given FL plus oxaliplatin and nine in the FL group had various other eligibilitycriteria violations.

\section{CHEMOTHERAPY}

The median number of cycles of chemotherapy received was 12 in both groups; 74.7 percent of patients in the group given FL plus oxaliplatin and 86.5 percent in the FL group received the planned 12 cycles. In the group that received FL plus oxaliplatin, the median dosage of oxaliplatin was 34.2 mg per square meter per week across all cycles received and $36.5 \mathrm{mg}$ per square meter per week across cycles including oxaliplatin. In both cases, more than 80 percent of the planned dose was actually given ( 80.5 percent and 85.9 percent, respectively). The dose of fluorouracil received was 84.4 percent of the planned dose in the group given FL plus oxaliplatin and 97.7 percent of the planned dose in the FL group.

\section{SAFETY}

Neutropenia, diarrhea, and vomiting were the most frequent grade 3 or 4 adverse effects in the group given FL plus oxaliplatin (Table 2). Grade 3 or 4 neutropenia was much commoner with FL plus oxaliplatin than with FL (41.1 percent vs. 4.7 percent, $\mathrm{P}<0.001$ ) but was complicated by fever or infection in only 1.8 percent of cases (20 patients) in the group given FL plus oxaliplatin and in 0.2 percent of cases ( 2 patients) in the FL group $(\mathrm{P}<0.001)$. The incidence of thromboembolic events among patients who received at least one cycle of the assigned regimen was similar in the two groups (63 of 1108 patients [5.7 percent] and 72 of 1111 patients [6.5 percent], respectively).

Although 92.1 percent of patients treated with FL plus oxaliplatin had peripheral neuropathy during treatment, half of these episodes were of grade 1 
OXALIPLATIN, FLUOROURACIL, AND LEUCOVORIN FOR COLON CANCER

\begin{tabular}{|c|c|c|c|c|c|c|c|c|}
\hline \multirow[t]{2}{*}{ Adverse Event } & \multicolumn{3}{|c|}{ FL plus Oxaliplatin $(\mathrm{N}=1108)$} & \multicolumn{3}{|c|}{$\mathrm{FL}(\mathrm{N}=1111)$} & \multicolumn{2}{|c|}{ P Value } \\
\hline & All Grades & Grade 3 & Grade 4 & All Grades & Grade 3 & Grade 4 & All Grades & Grades 3 and 4 \\
\hline \multicolumn{9}{|c|}{ percent } \\
\hline Paresthesia† & 92.0 & 12.4 & NA & 15.6 & 0.2 & NA & $<0.001$ & 0.001 \\
\hline Neutropenia & 78.9 & 28.8 & 12.3 & 39.9 & 3.7 & 1.0 & $<0.001$ & $<0.001$ \\
\hline Thrombocytopenia & 77.4 & 1.5 & 0.2 & 19.0 & 0.2 & 0.2 & $<0.001$ & 0.001 \\
\hline Anemia & 75.6 & 0.7 & 0.1 & 66.9 & 0.3 & 0.0 & $<0.001$ & 0.09 \\
\hline Nausea & 73.7 & 4.8 & 0.3 & 61.1 & 1.5 & 0.3 & $<0.001$ & $<0.001$ \\
\hline Diarrhea & 56.3 & 8.3 & 2.5 & 48.4 & 5.1 & 1.5 & $<0.001$ & $<0.001$ \\
\hline Vomiting & 47.2 & 5.3 & 0.5 & 24.0 & 0.9 & 0.5 & $<0.001$ & $<0.001$ \\
\hline Stomatitis & 41.6 & 2.7 & 0.0 & 39.6 & 2.0 & 0.2 & 0.34 & 0.41 \\
\hline Skint & 31.5 & 1.4 & 0.6 & 35.5 & 1.7 & 0.7 & 0.05 & 0.67 \\
\hline Alopecia』 & 30.2 & NA & NA & 28.1 & NA & NA & 0.28 & NA \\
\hline Allergic reaction & 10.3 & 2.3 & 0.6 & 1.9 & 0.1 & 0.1 & $<0.001$ & $<0.001$ \\
\hline $\begin{array}{l}\text { Thrombosis } \\
\text { or phlebitis }\end{array}$ & 5.7 & 1.0 & 0.2 & 6.5 & 1.7 & 0.1 & 0.48 & 0.29 \\
\hline $\begin{array}{l}\text { Neutropenia with } \\
\text { fever or infection }\end{array}$ & 1.8 & 1.4 & 0.4 & 0.2 & 0.1 & 0.1 & $<0.001$ & $<0.001$ \\
\hline
\end{tabular}

* Fisher's exact test was used to calculate P values. NA denotes not applicable.

$\dagger$ There are only three grades of paresthesia in version 1 of the Common Toxicity Criteria of the National Cancer Institute.

This category included the hand-foot syndrome.

$\int$ There are only two grades of alopecia in version 1 of the Common Toxicity Criteria of the National Cancer Institute. The incidence of grade 2 alopecia was 5.0 percent in each group.

(Table 3). Of the 137 patients (12.4 percent) who had grade 3 peripheral neuropathy during treatment, grade 3 symptoms were still present in 8 patients at the six-month follow-up visit and in 5 patients at the one-year visit. In 12 patients, grade 3 peripheral neurosensory symptoms appeared after the end of treatment, and 6 of these patients had persistent grade 3 symptoms after one year. In total, 11 of 1018 patients (1.1 percent) who were assessed one year after the end of treatment continued to have grade 3 peripheral neurosensory symptoms. This number dropped to five ( 0.5 percent) after 18 months (Table 3).

Twelve patients - six in each group ( 0.5 percent) - died within 1 month after the end of treatment; these included three deaths in each group during the first 60 days of treatment. In the group given FL plus oxaliplatin, four patients died of infection or sepsis (two with neutropenia) and two of intracranial hemorrhage. In the FL group, one patient each died of sepsis, Stevens-Johnson syndrome in the context of severe diarrhea and fluconazole treatment, and anoxic cerebral infarction; one patient committed suicide; and two died suddenly from cardiac causes.
FOLLOW-UP

There was good compliance with follow-up visits. The mean time between visits was 5.97 months in the group given FL plus oxaliplatin and 5.98 months in the FL group. The median interval was 6.01 months in both groups.

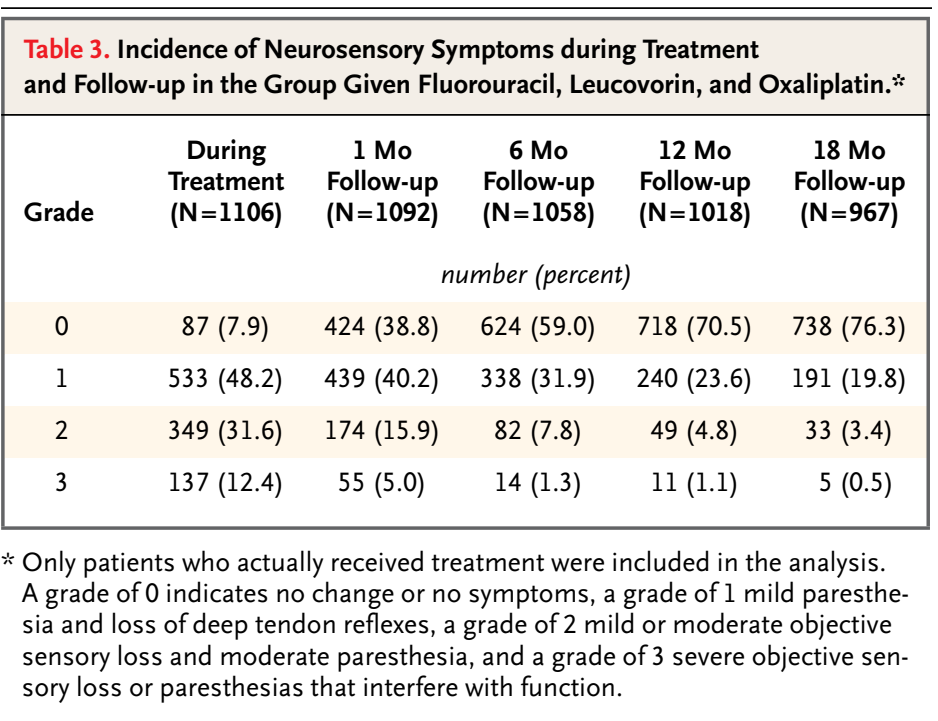


DISEASE-FREE SURVIVAL

At the time of analysis (median follow-up, 37.9 months), 237 patients in the group given FL plus oxaliplatin (21.1 percent) had relapsed or died, as compared with 293 (26.1 percent) in the FL group.

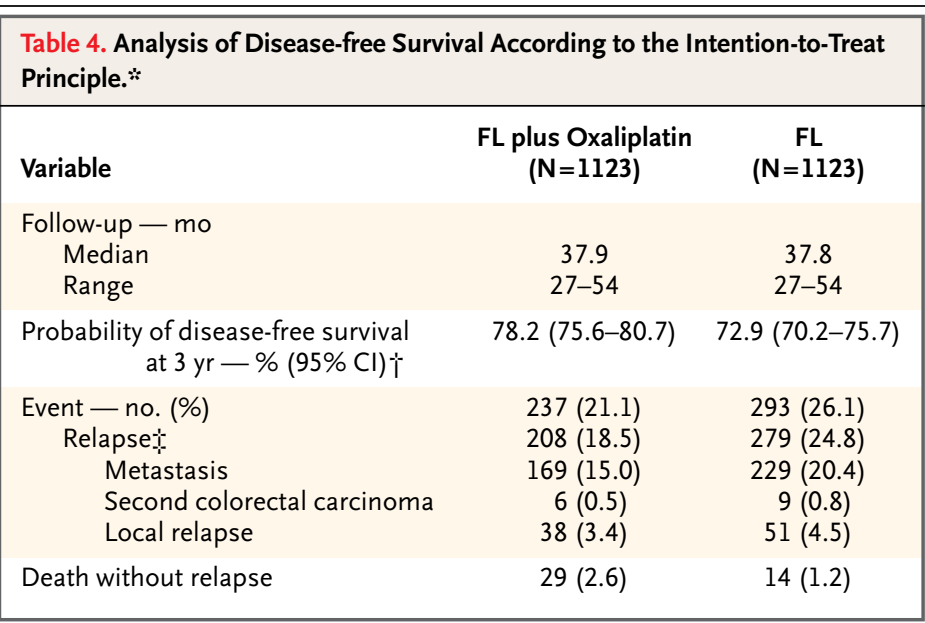

* FL denotes fluorouracil and leucovorin, and $\mathrm{Cl}$ confidence interval. $\dagger \mathrm{P}=0.002$ by the stratified log-rank test for the comparison between groups. $\checkmark$ The same patient could have been counted in more than one relapse category if several types of relapses were reported at the same follow-up visit.

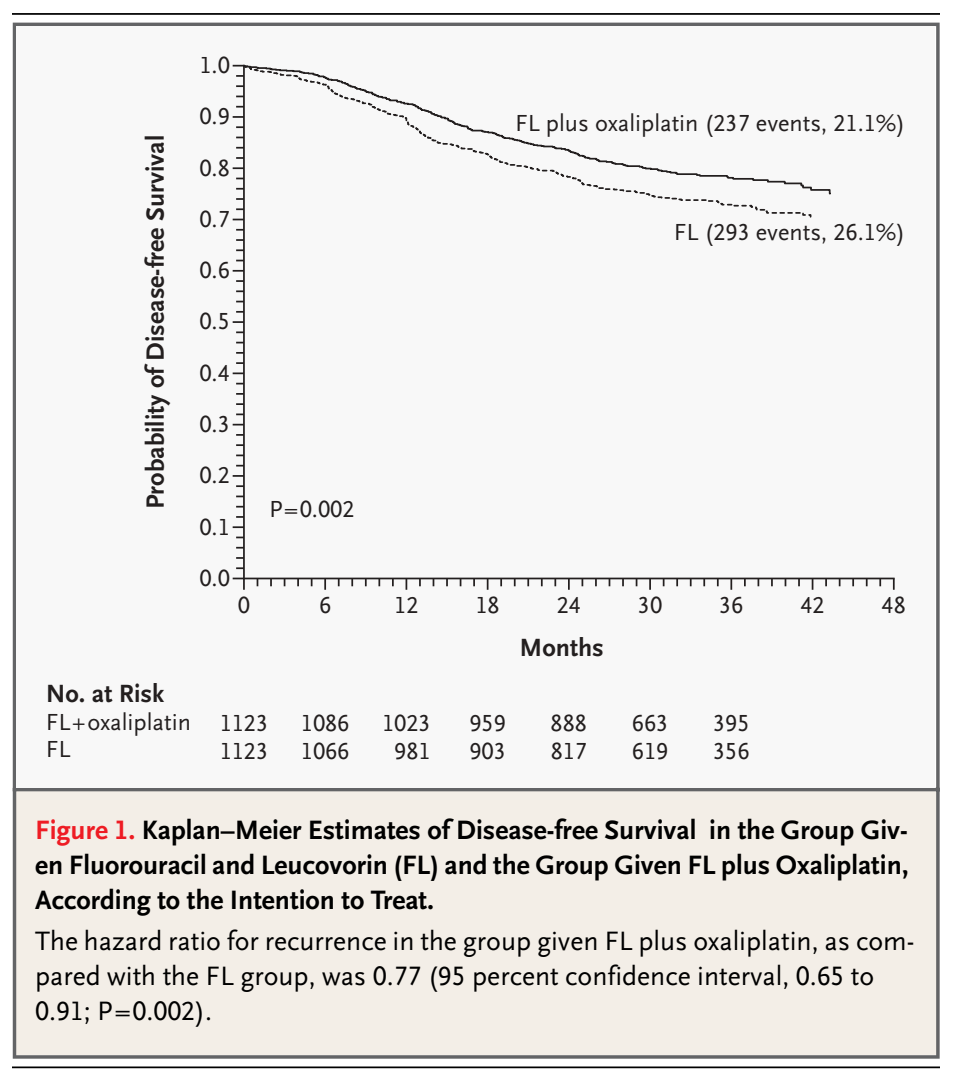

The hazard ratio for recurrence in the group given FL plus oxaliplatin, as compared with the FL group, was $0.77(\mathrm{P}=0.002)$, corresponding to a 23 percent reduction in the risk of relapse. The probability of disease-free survival at three years was 78.2 percent (95 percent confidence interval, 75.6 to 80.7 percent) in the group given FL plus oxaliplatin and 72.9 percent ( 95 percent confidence interval, 70.2 to 75.7 percent) in the FL group ( $\mathrm{P}=0.002$ by the stratified log-rank test) (Table 4 and Fig. 1).

Among patients with stage III disease, the hazard ratio for relapse was 0.76 (95 percent confidence interval, 0.62 to 0.92 ) in the group given $\mathrm{FL}$ plus oxaliplatin, as compared with the FL group, and the three-year disease-free survival rate was 72.2 percent and 65.3 percent, respectively (Fig. 2). Among patients with stage II disease, the hazard ratio for relapse was 0.80 (95 percent confidence interval, 0.56 to 1.15 ) in the group given FL plus oxaliplatin, as compared with the FL group, and the three-year disease-free survival rates were 87.0 percent and 84.3 percent, respectively (Fig. 2).

A Cox-model analysis showed that the reduced risk of recurrence with FL plus oxaliplatin was similar in patients with stage II and those with stage III disease $(\mathrm{P}=0.77)$. Calculation of hazard ratios and 95 percent confidence intervals (Fig. 3) showed that the reduced risk of relapse was consistent in all subgroups defined on the basis of prognostic factors at baseline.

\section{OVERALL SURVIVAL}

At the time of the cutoff date of the primary analysis, 133 patients had died in the group given FL plus oxaliplatin, as compared with 146 patients in the FL group (hazard ratio for death, 0.90 ; 95 percent confidence interval, 0.71 to 1.13 ), and the probability of survival at three years was 87.7 percent and 86.6 percent, respectively. Most of the patients who died had stage III disease (104 in the group given FL plus oxaliplatin and 119 in the FL group); the hazard ratio for death in this subgroup was 0.86 (95 percent confidence interval, 0.66 to 1.11 ).

\section{I SCUSSION}

In previous trials, the addition of oxaliplatin to fluorouracil and leucovorin doubled the response rate and prolonged progression-free survival among patients with metastatic colorectal cancer. ${ }^{14}$ The efficacy and safety of this regimen were recently confirmed in a large, randomized, phase 3 trial, 


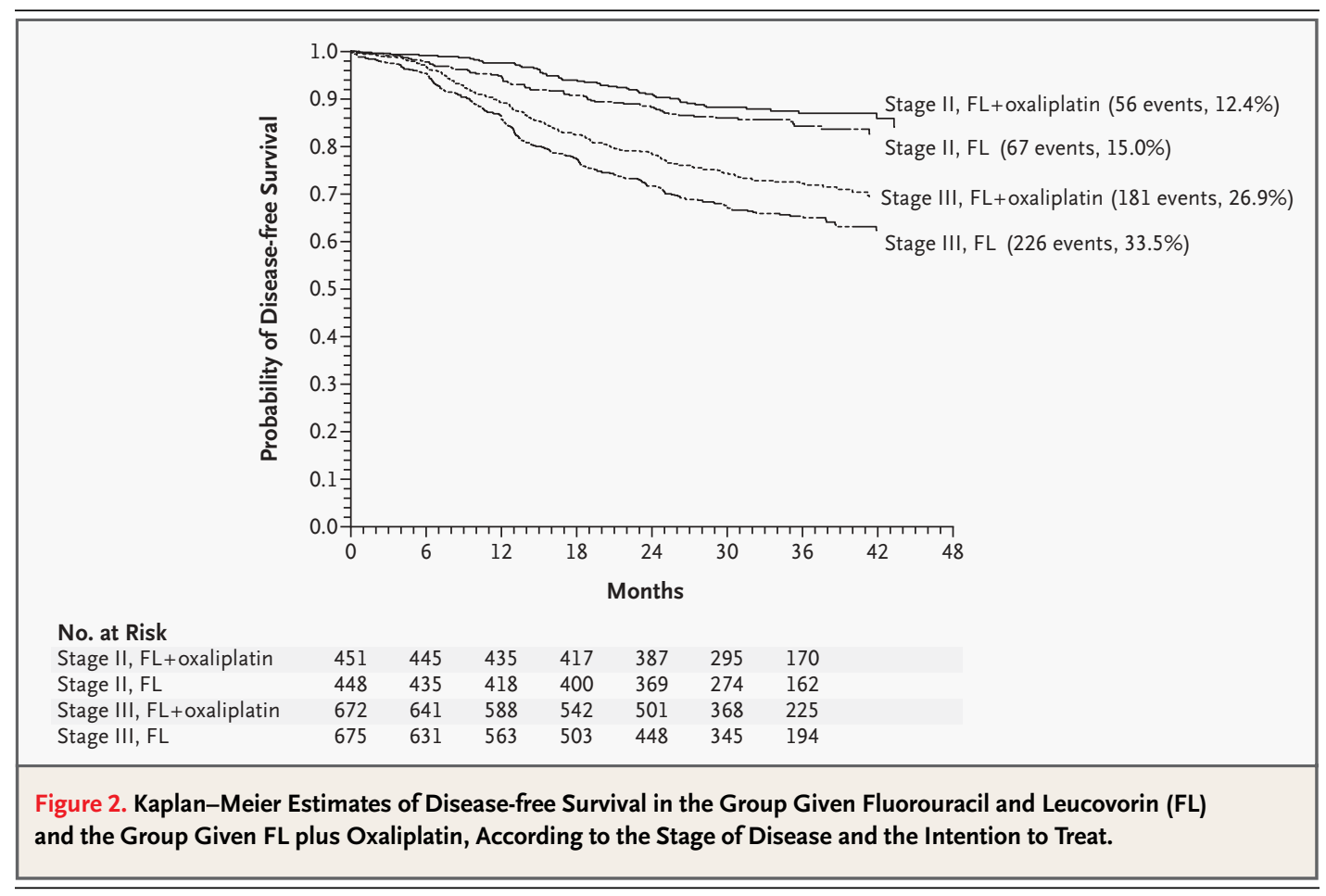

which found that this approach was superior (with respect to all efficacy variables, including overall survival) to the combination of irinotecan, fluorouracil (given as a bolus), and leucovorin. ${ }^{15}$ Our trial was designed to test the efficacy of adjuvant treatment with the regimen of FL plus oxaliplatin. We chose disease-free survival as the primary end point of the study because, like others ${ }^{16}$ we believe that the absence of relapse is the best indicator of efficacy, since it relates directly to the effect of the treatment under investigation. By allowing early appraisal of the results, the use of three-year disease-free survival as the primary end point for adjuvant trials of patients with colon cancer should permit rapid evaluation of new treatments. Whether disease-free survival should be a primary end point is still under discussion, but a recent analysis of several studies supports the appropriateness of the use of three-year disease-free survival as a good predictor of five-year overall survival in trials of adjuvant treatment of colon cancer. ${ }^{17}$

Disease-free survival in the FL group in our study falls within the highest range reported in most studies of adjuvant treatment of colon cancer with FL. ${ }^{6-8,18,19}$ The improvement in disease-free survival among patients who were treated with FL plus oxaliplatin corresponds to a relative reduction in the risk of recurrence of 23 percent. Since most relapses after curative surgery occur within the first three years, we consider our results in this respect to be complete.

Although it is agreed that patients with stage III disease benefit from adjuvant treatment, whether all patients with stage II disease should receive such treatment remains debatable. This controversy was sustained for years by the contradictory conclusions of two large groups of investigators. The National Surgical Adjuvant Breast and Bowel Project concluded that the relative benefits of treatment were largely the same for stage II and stage III tumors, ${ }^{20}$ whereas the International Multicentre Pooled Analysis of B2 Colon Cancer Trials (IMPACT B2) failed to demonstrate a statistically significant benefit for stage II tumors. ${ }^{21}$

A recent meta-analysis from the Mayo Clinic, ${ }^{22}$ which evaluated individual data on 3300 patients who were enrolled in five randomized trials, including those analyzed in IMPACT B2, concluded that patients with stage II disease could benefit from adjuvant chemotherapy, but to a lesser extent than patients with stage III tumors. Indeed, the absolute benefit among patients with stage II disease is only half as great as that among patients with stage III disease, and twice as many patients with stage II tumors are required in such studies in order to detect a difference. 
A test for interaction is an appropriate statistical approach to the question of whether the benefit of adjuvant treatment differs between stage II and stage III colorectal cancer. ${ }^{23}$ In our study, this test showed no significant interaction between the stage of disease and the treatment, indicating that FL plus oxaliplatin benefited both stage II and stage

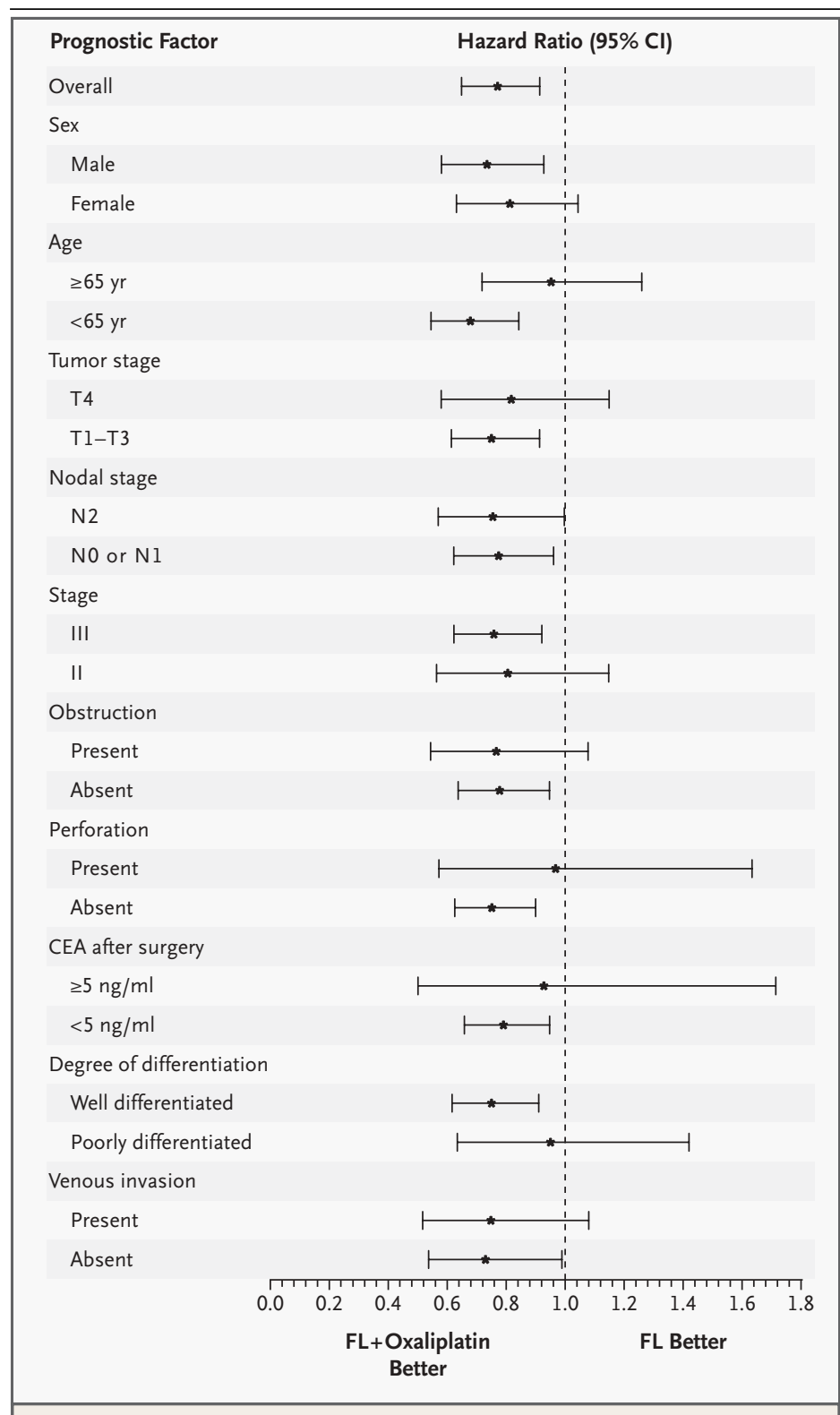

Figure 3. Hazard Ratios and 95 Percent Confidence Intervals for Recurrence in the Group Given Fluorouracil and Leucovorin (FL) plus Oxaliplatin, as Compared with the FL Group, According to Baseline Prognostic Factors and the Intention to Treat.

CEA denotes carcinoembryonic antigen.
III colorectal cancer. From a clinical standpoint, stage II colon cancer occurs in a heterogeneous, node-negative population in which clinical and biologic prognostic factors other than the status of lymph-node involvement need to be taken into account. Tools are being developed to help physicians assess the risk-benefit ratio of adjuvant chemotherapies for individual patients. ${ }^{22}$

With no clear consensus on the most effective FL regimen to be used for adjuvant treatment, we chose a twice-monthly regimen because of its efficacy and low rates of adverse effects in patients with advanced colorectal cancer, alone and in combination with oxaliplatin. ${ }^{12,14,24}$ Supporting our decision are recent results demonstrating that this approach to adjuvant therapy is less toxic than monthly bolus injections of FL and is just as effective. ${ }^{18}$ This approach led to a straightforward study design, since the treatment in both groups was similar except for the addition of oxaliplatin in the group given FL plus oxaliplatin. The improved disease-free survival in the FL-plus-oxaliplatin group is thus directly linked to oxaliplatin.

The main safety concern regarding the use of oxaliplatin is peripheral neuropathy. Oxaliplatin induces frequent, transient, distal paresthesias during or shortly after the first minutes of infusion. In some cases these neurosensory symptoms increase in intensity with cumulative doses, persist between cycles, and interfere with function (in the case of grade 3 effects). ${ }^{12,14,25}$ We observed grade 3 peripheral neuropathy in 12.4 percent of patients who were receiving oxaliplatin. At one year, 11 patients (1.1 percent) had grade 3 neuropathy. Among them, two were found to have underlying disease that could have caused these symptoms (diabetes and hemiplegia, respectively). Although more frequent among patients receiving FL plus oxaliplatin than among those treated with FL alone, grade 3 or 4 neutropenia led to fever or infection in only 1.8 percent of patients in the former group. Similar findings have been reported among patients with metastatic colorectal cancer. ${ }^{14}$ From a safety standpoint, the rate of death from any cause was similarly low during treatment in both groups and, at 0.5 percent, is among the lowest figures reported in trials of adjuvant chemotherapy. $5,18,26,27$

Figures for overall survival at this stage of the study are preliminary, and no conclusion can be drawn about differences in survival between the treatment groups. Since the median overall survival from the time of diagnosis of metastatic colorec- 
tal cancer is approximately 20 months, ${ }^{13-15,28}$ we expect that the effect of oxaliplatin on survival will become apparent within the next 2 years.

Supported by Sanofi-Synthelabo.

Dr. Boni reports having received consulting fees from SanofiSynthelabo and Lilly; Dr. Tabernero consulting and lecture fees from Sanofi-Synthelabo; and Dr. Hickish consulting and lecture fees from Sanofi-Synthelabo, Aventis, and Roche. Dr. Topham reports having equity ownership in Sanofi-Synthelabo and having received lecture fees from Sanofi-Synthelabo and consulting fees from Roche and Baxter. Dr. Bridgewater reports having received consulting fees from Novartis Consumer Health; Dr. Clingan consulting fees from SanofiSynthelabo and Roche Australia; and Dr. de Gramont consulting and lecture fees from Sanofi-Synthelabo and Baxter. Dr. MounedjiBoudiaf and Dr. Tabah-Fisch are employed by Sanofi-Synthelabo.

We are indebted to all those who have contributed to the conduct and analysis of the MOSAIC trial, including the 146 investigators from France, the United Kingdom, Spain, Italy, Belgium, Greece, Hungary, the Netherlands, Portugal, Germany, Sweden, Austria, Poland, Denmark, Norway, Australia, Israel, Cyprus, Singapore, and Switzerland who enrolled patients in the study; to the members of the data and safety monitoring board, especially Marc Buyse (statistician); to the dedicated teams from Sanofi-Synthelabo, including Christelle Lorenzato and Robert Bigelow (statistical department) and Jeanne Marceau-Suissa, Nathalie Lebail, and Noelle Muller (clinical development); and to Daniel Sargent (Mayo Clinic) for the discussion of the results.
REFERENCES

1. GLOBOCAN 2000: cancer incidence, mortality and prevalence worldwide, version 1.0. IARC CancerBase no. 5. Lyon, France: IARC Press, 2001.

2. Obrand DI, Gordon PH. Incidence and patterns of recurrence following curative resection for colorectal carcinoma. Dis Colon Rectum 1997;40:15-24.

3. Colon and rectum. In: American Joint Committee on Cancer. AJCC cancer staging manual. 5th ed. Philadelphia: LippincottRaven, 1997:83-90.

4. Moertel CG, Fleming TR, Macdonald JS et al. Levamisole and fluorouracil as adjuvant therapy of resected colon carcinoma. N Engl J Med 1990;322:352-8.

5. Wolmark N, Rockette H, Fisher B, et al. The benefit of leucovorin-modulated fluorouracil as postoperative adjuvant therapy for primary colon cancer: results from National Surgical Adjuvant Breast and Bowel Project protocol C-03. J Clin Oncol 1993;11 1879-87.

6. International Multicentre Pooled Analysis of Colon Cancer Trials (IMPACT) Investigators. Efficacy of adjuvant fluorouracil and folinic acid in colon cancer. Lancet 1995 345:939-44.

7. O'Connell MJ, Mailliard JA, Kahn MJ, et al. Controlled trial of fluorouracil and lowdose leucovorin given for 6 months as postoperative adjuvant therapy for colon cancer. J Clin Oncol 1997;15:246-50.

8. Wolmark N, Rockette H, Mamounas E, et al. Clinical trial to assess the relative efficacy of fluorouracil and leucovorin, fluorouracil and levamisole, and fluorouracil, leucovorin, and levamisole in patients with Dukes' B and C carcinoma of the colon: results from National Surgical Adjuvan Breast and Bowel Project C-04. J Clin Oncol 1999; 17:3553-9.

9. O'Connell MJ, Laurie JA, Kahn M, et al. Prospectively randomized trial of postoperative adjuvant chemotherapy in patients with high-risk colon cancer. J Clin Oncol 1998 16:295-300.

10. Haller DG, Catalano PJ, Macdonald JS, Mayer RJ. Fluorouracil (FU), leucovorin (LV) and levamisole (LEV) adjuvant therapy for colon cancer: five-year final report of INT-
0089. Prog Proc Am Soc Clin Oncol 1998; 17:256a.

11. Zaniboni A. Adjuvant chemotherapy in colorectal cancer with high-dose leucovorin and fluorouracil: impact on disease-free survival and overall survival. J Clin Oncol 1997 15:2432-41.

12. Andre $T$, Bensmaine $M A$, Louvet $C$, et al. Multicenter phase II study of bimonthly highdose leucovorin fluorouracil infusion, and oxaliplatin for metastatic colorectal cance resistant to the same leucovorin and fluorouracil regimen. J Clin Oncol 1999;17:3560-8. 13. Giacchetti S, Perpoint B, Zidani R, et al. Phase III multicenter randomized trial of oxaliplatin added to chronomodulated fluorouracil-leucovorin as first-line treatment of metastatic colorectal cancer. J Clin Onco 2000;18:136-47.

14. de Gramont A, Figer A, Seymour M, et al. Leucovorin and fluorouracil with or without oxaliplatin as first-line treatment in advanced colorectal cancer. J Clin Oncol 2000;18:2938-47.

15. Goldberg RM, Sargent DJ, Morton RF et al. A randomized controlled trial of fluorouracil plus leucovorin, irinotecan and oxaliplatin combinations in patients with previously untreated metastatic colorectal cancer. J Clin Oncol 2004;22:23-30.

16. Elfenbein GJ. Stem-cell transplantation for high-risk breast cancer. N Engl J Med 2003;349:80-2.

17. Sargent D, Wieand S, Goldberg R, et al 3 Year DFS vs. 5 year OS as an endpoint for adjuvant colon cancer studies: data from randomized trials. Rockville, Md.: Food and Drug Administration, November 2003. (Accessed May 10, 2004, at http://www.fda gov/cder/drug/cancer_endpoints/Sargent/ index.htm.)

18. Andre T, Colin P, Louvet C, et al. Semimonthly versus monthly regimen of fluorouracil and leucovorin administered for 24 or 36 weeks as adjuvant therapy in stage II and III colon cancer: results of a randomized trial. J Clin Oncol 2003;21:2896-903.

19. Francini G, Petrioli R, Lorenzini L, et al. Folinic acid and 5-fluorouracil as adjuvant chemotherapy in colon cancer. Gastroenterology 1994;106:899-906.
20. Mamounas E, Wieand S, Wolmark N, et al. Comparative efficacy of adjuvant chemotherapy in patients with Dukes' B versus Dukes' C colon cancer: results from four National Surgical Adjuvant Breast and Bowel Project adjuvant studies (C-01, C-02, C-03, and C-04). J Clin Oncol 1999;17:1349-55.

21. International Multicentre Pooled Analysis of B2 Colon Cancer Trials (IMPACT B2) Investigators. Efficacy of adjuvant fluorouracil and folinic acid in B2 colon cancer. J Clin Oncol 1999;17:1356-63.

22. Gill S, Loprinzi CL, Sargent DJ, et al. Using a pooled analysis to improve the understanding of adjuvant therapy (AT) benefit for colon cancer (CC). Prog Proc Am Soc Clin Oncol 2003;22:253a.

23. Buyse M, Piedbois P. Should Dukes' B patients receive adjuvant therapy? A statistical perspective. Semin Oncol 2001;28:Suppl 1:20-4.

24. de Gramont A, Bosset JF, Milan C, et al. Randomized trial comparing monthly lowdose leucovorin and fluorouracil bolus with bimonthly high-dose leucovorin and fluorouracil bolus plus continuous infusion for advanced colorectal cancer: a French intergroup study. J Clin Oncol 1997;15:808-15. 25. Extra JM, Espie M, Calvo F, Ferme C, Mignot L, Marty M. Phase I study of oxaliplatin in patients with advanced cancer. Cancer Chemother Pharmacol 1990;25:299-303. 26. Rothenberg ML, Meropol NJ, Poplin EA, Van Cutsem E, Wadler S. Mortality associated with irinotecan plus bolus fluorouracil/leucovorin: summary findings of an independent panel. J Clin Oncol 2001;19:3801-7. 27. Wolmark N, Bryant J, Smith R, et al. Adjuvant 5-fluorouracil and leucovorin with or without interferon alfa-2a in colon carcinoma: National Surgical Adjuvant Breast and Bowel Project protocol C-05. J Natl Cancer Inst 1998;90:1810-6.

28. Tournigand $\mathrm{C}$, André $\mathrm{T}$, Achille $\mathrm{E}$, et al. FOLFIRI followed by FOLFOX 6 or the reverse sequence in advanced colorectal cancer: a randomized GERCOR study. J Clin Oncol 2004;22:229-37.

Copyright (c) 2004 Massachusetts Medical Society. 